Neuropharmacology and Analgesia

\title{
An in vitro model for testing drugs to treat tinnitus
}

\author{
Calvin Wu ${ }^{\mathrm{a}, \mathrm{b}, \mathrm{c}}$, Kamakshi Gopal ${ }^{\mathrm{b}, \mathrm{c}}$, Guenter W. Gross ${ }^{\mathrm{a}, \mathrm{c}}$, Thomas J. Lukas ${ }^{\mathrm{d}}$, Ernest J. Moore ${ }^{\mathrm{b}, \mathrm{c}, \mathrm{d}, *}$ \\ a Department of Biology, University of North Texas, Denton, TX 76203, USA \\ ${ }^{\mathrm{b}}$ Department of Speech \&' Hearing Sciences, University of North Texas, Denton, TX 76203, USA \\ c Center for Network Neuroscience, University of North Texas, Denton, TX 76203, USA \\ ' Department of Molecular Pharmacology E Biological Chemistry, Feinberg School of Medicine, Northwestern University, Chicago, IL 60611, USA
}

\section{A R T I C L E I N F O}

\section{Article history:}

Received 8 February 2011

Received in revised form 16 May 2011

Accepted 22 May 2011

Available online 2 June 2011

\section{Keywords:}

Tinnitus

Pentylenetetrazol (PTZ)

Linopirdine

L-Carnitine

Pregabalin

Gabapentin

Drug repositioning

\begin{abstract}
A B S T R A C T
Tinnitus affects approximately 50 million people in the USA alone, with 10 million being highly debilitated. Pharmacotherapy for tinnitus is still in emerging stages due to time consuming clinical trials and/or animal experiments. We tested a new cellular model where induced rapid neuronal firing or spiking was used as a mimic for the type of aberrant activity that may occur in tinnitus. Spontaneously active auditory cortical networks growing on microelectrode arrays were exposed to pentylenetetrazol (PTZ), a proconvulsant and an antagonist of $\mathrm{GABA}_{\mathrm{A}}$ receptor, which is implicated in tinnitus. Auditory cortical networks were then exposed to experimental tinnitus drugs linopirdine (Dup966, a potassium channel blocker), L-carnitine (an antioxidant), or selective $\mathrm{Ca}^{2+}$ channel antagonists pregabalin (Lyrica), or gabapentin (Neurontin) at various concentrations. PTZ increased spike rate by $139.6 \pm 27 \%$ and burst rate by $129.7 \pm 28 \%$ in auditory cortical networks with a phenotypic high firing of excitable neurons. Reductions of increased activity were observed to varying degrees using the experimental tinnitus drugs. The potency of the drugs was linopirdine $\left(\mathrm{EC}_{50}: 176 \pm 7.0 \mu \mathrm{M}\right)>\mathrm{L}_{\text {-carnitine }}\left(\mathrm{EC}_{50}\right.$ : $1569 \pm 41 \mu \mathrm{M})>$ pregabalin $\left(\mathrm{EC}_{50}: 8360 \pm 340 \mu \mathrm{M}\right)$, > gabapentin, with $34.2 \pm 7.5 \%$ efficacy $\left(\mathrm{EC}_{50}: 2092 \pm\right.$ $980 \mu \mathrm{M})$. These studies provide proof of principle for the use of auditory cortical networks on microelectrode array as a feasible platform for semi-high throughput application for screening of drugs that might be used for the treatment of tinnitus.
\end{abstract}

(C) 2011 Elsevier B.V. All rights reserved.

\section{Introduction}

Noises or ringing in the ears are experienced by people with normal hearing (Thabet, 2009), but most noticeable by individuals who have some type of auditory deficit (Littler, 1965). The malady is known as tinnitus, and affects approximately 50 million people in the USA alone. The sounds perceived are usually high-pitched whistles, but some individuals report hearing other noises such as hissing, buzzing, chirping, clicking, or other internally generated annoying sounds. The basic underlying mechanisms responsible for the generation of tinnitus after otologic insult are largely unknown (Jung et al., 1993; Passe, 1951). While various anatomical structures of the auditory system may be the underlying site of tinnitus generation, the hair cells of the inner ear cochlea may be the most vulnerable structures. One school of thought about the genesis of tinnitus is that the hair cell releases an unusually high amount of neurotransmitter substances such as glutamate, which excites afferent fibers of the VIIIth cranial nerve (Zenner and Ernst, 2003). This over excitation is perceived as a phantom sound (Denk et al., 1997). However, tinnitus may also have its origin in the brainstem or cortex, and thus, the unwanted sounds are perceived

* Corresponding author at: Dept. of Speech \& Hearing Sciences, University of North Texas, Denton, TX 76203-5010, USA. Tel.: +1 940565 2481; fax: + 19405654058.

E-mail address: ejmoore@unt.edu (E.J. Moore). also to be originating in the brain (Lockwood et al., 1999; Mühlau et al., 2006). The sensory neural axis of the auditory system (Jastreboff, 1990) such as the dorsal cochlear nucleus (Kaltenbach, 2000, 2006) has been similarly indicated. Nevertheless, the basic mechanisms underlying tinnitus that might lead to better treatment regimens are not well understood and have not been readily forthcoming (Kay and Davies, 1993; Lanting et al., 2009).

Tinnitus is a highly debilitating disorder for many patients and the condition is exacerbated by the fact that no chemotherapeutic agents have been found to alleviate the problem over a long period of time (Baguley, 2002). The problem lies with the fact that the fundamental physiological and biochemical mechanism or mechanisms underlying tinnitus remain unclear (Kaltenbach, 2000, 2006). It would be of great benefit to millions of tinnitus patients if effective pharmaceutical agents could be found to provide relief for tinnitus. Since pharmaceutical product development requires $10-15$ years and at costs estimated between 500 million and 2.0 billion dollars to bring a drug to market, the concept of "repurposing" or "repositioning" of FDA approved drugs has been suggested (Boguski et al., 2009; Tobinick, 2009). However, a suitable screening assay is necessary to find drugs to treat tinnitus before repurposing.

Eggermont and colleagues hypothesized that an increase in neuronal firing may be indicative of tinnitus of auditory cortical neuronal origin (Eggermont, 2008; Kenmochi and Eggermont, 1997). The central origin 
is widely speculated also to be due to its role in changing neural plasticity (Lockwood et al., 1998, 2002). We used in vitro auditory cortical networks (collections of neurons) derived from mouse embryos, growing on microelectrode arrays as a platform to induce rapid neuronal firing to mimic aberrant activity that may be similar to tinnitus. The proconvulsant drug pentylenetetrazol (PTZ) was applied to the neuronal networks to induce an increase in neuronal firing rate. Four "experimental tinnitus drugs" (pregabalin, gabapentin, linopirdine, L-carnitine) were applied at various concentrations to test their efficacy to reduce the rapid neuronal firing that was hypothesized to mimic the excitatory activity that may occur in tinnitus.

Pentylenetetrazol [6,7,8,9-tetrahydro-5H-tetrazolo(1,5-a)azepine] $\left(\mathrm{C}_{6} \mathrm{H}_{10} \mathrm{~N}_{4}\right)$ (Pentrazol) is a respiratory and circulatory stimulant that in high doses causes convulsions - its mechanism of action is a GABA antagonist. Pregabalin [(S)-3-(aminomethyl)-5-methylhexanoic acid] $\left(\mathrm{C}_{8} \mathrm{H}_{17} \mathrm{NO}_{2}\right)$ (Lyrica) is an anticonvulsant drug that is used for neuropathic pain, partial seizures and generalized anxiety disorder. The mechanism of action is a calcium channel blocker or a GABA agonist. Gabapentin [2-[1-(aminomethyl) cyclohexyl] acetic acid] $\left(\mathrm{C}_{9} \mathrm{H}_{17} \mathrm{NO}_{2}\right)$ (Neurontin) was originally thought to be a GABA analog and a treatment for epilepsy, although subsequently it was determined that the former was not substantiated. It was originally developed for use as an adjunctive therapy for epilepsy, but presently is used for neuropathic pain and major depressive disorder. Linopirdine [1-phenyl-3,3-bis(pyridin-4ylmethyl)-1,3-dihydro-2H-indol-2-one] $\left(\mathrm{C}_{26} \mathrm{H}_{21} \mathrm{~N}_{3} \mathrm{O}\right)$ (linopirdine) is a psychostimulant/nootropic drug that acts as a potassium channel blocker, and releases acetylcholine. L-Carnitine (3-hydroxy-4-(trimethylazaniumyl)butanoate) $\left(\mathrm{C}_{7} \mathrm{H}_{15} \mathrm{NO}_{3}\right)$ (carnitine) is a quaternary ammonium compound that is biosynthesized from the amino acids lysine and methionine. One mechanism of action is thought to be an antioxidant within the mitochondria of cells.

Here we present the first observation of the use of the proconvulsant drug, PTZ (Klöcker et al., 1996), to excite mouse neuronal cortical activity recorded in vitro, as a homologue for tinnitus. We further investigated the modulation of the excitatory neuronal activity by drugs of the class: Ca-channel blocker, K-channel blocker, or antioxidant. These drugs exhibited differential effects of reducing over-excitation of neuronal activity and thus suggest a possible chemotherapeutic treatment for tinnitus.

\section{Methods}

\subsection{Isolation of murine auditory cortex}

The care and use of animals, as well as all procedures involving animals in this study were approved by and conducted in accordance with the guideline of the institutional animal care and use committee. Mouse embryos (Balb-C/ICR, day E17) were extracted from the dame after $\mathrm{CO}_{2}$ narcosis and cervical dislocation, followed by a double thoracotomy. Auditory cortices located on the posterolateral surface and depths of the left temporal cortex (TE1, TE2 and TE3) were dissected $(\sim 2 \times 2 \times 1 \mathrm{~mm})$ from the embryos under sterile conditions.

\subsection{Cell culture and microelectrode array fabrication}

The standard culturing process published earlier was used in this study (Gopal and Gross, 1996; Keefer et al., 2001). Briefly, the auditory cortical cells were mechanically minced, enzymatically dissociated, triturated and mixed with Dulbecco's Modified Minimal Essential Medium (DMEM). Horse serum (10\%) and fetal bovine serum (10\%) were added to the DMEM. The cell suspension was decorated onto the microelectrode array surface decorated with poly-D-lysine and laminin and incubated at $37^{\circ} \mathrm{C}$ in a $10 \% \mathrm{CO}_{2}, 90 \%$ air atmosphere. The age of cultures used in this set of experiments ranged from 21 to 96 days in vitro with an average age of $31 \pm 2$ days. Fabrication and preparation of microelectrode arrays, as well as recording techniques have been described previously (Gopal and Gross, 2004; Keefer et al., 2001).

\subsection{Solutions and drugs}

Drug solutions were made on the day of experiments from stock solutions. Solutions and drug applications were made to a constant volume of $1.0-2.0 \mathrm{ml}$ of the experimental bath. Drug concentrations were calculated to minimize bulk volume changes to the experimental bath. Pentylenetetrazol, gabapentin, linopirdine and L-carnitine were obtained from Sigma-Aldrich (St. Louis, MO), while pregabalin was obtained from Pfizer Pharmaceutical Company (Groton, CT).

\subsection{Electrophysiological recordings and analyses of microelectrode data}

Microelectrode arrays were placed in a stainless steel recording chamber mounted on an inverted microscope head stage and maintained at $37^{\circ} \mathrm{C}$. Chamber components were sterilized via autoclaving before each experiment. The original medium was replaced by fresh DMEM stock medium (without serum). The $\mathrm{pH}$ was maintained at 7.4 with a continuous stream of filtered, humidified, $10 \% \mathrm{CO}_{2}$ confined by a cap with a heated ITD window to prevent condensation and allow continuous microscopic observation. Evaporation due to constant air flow was compensated by a syringe infusion pump with sterile water. Auditory neuronal activity was recorded with a two-stage, 64-channel amplifier system (Plexon, Dallas, TX), and digitized simultaneously at $40 \mathrm{kHz}$ via a Dell 8600 workstation. Total system gain was set to $1.2 \times 10^{3}$. Spike identification and separation was accomplished with a real time template-matching algorithm (Plexon, Dallas, TX) to provide single-unit spike rate data. Electrodes/channels were assigned to 64 digital signal processors. Each digital signal processor can discriminate up to four different action potential waveforms or "active" units. Multiple unit data were analyzed off-line using custom programs for burst recognition and analyses (Gopal et al., 2007). Burst patterns derived from spike integration ( $\tau=100 \mathrm{~ms}$ ) provided a high signal-to-noise feature extraction that has been shown previously to reveal several modes of neuronal network activity in the form of action potentials (spike activity) of high frequency clusters (burst activity). From this approach, single unit activity was averaged to yield spike rate and burst rate, readily computed from individual recording sites. All data presented are based on quantification of single unit parameters. Data obtained were analyzed using custom and commercially available data analyses software programs (Plexon, Dallas, TX). The primary data display was the real time plotting of total or average network spike production per minute.

\subsection{Data analyses}

In order to quantify neuronal network responses, the mean spike and mean burst rates were used. One-minute average activity across all discriminated units was derived for each experiment. Bursts were identified for each discriminated unit by integration (Morefield et al., 2000). Burst rates depicted in figures represent an average of all bursts per minute. In order to control for differences in the spontaneous activity among cultures, the PTZ-induced activity was quantified as a percent change from the reference (DMEM stock) activity. Furthermore, tinnitus drug-induced activity was quantified as a percent change from the PTZ-induced activity. Waveform analysis was performed by comparing peak width (P1-P3) and amplitude (P1-P2 and P2-P3) from a total of 10 waveforms. Statistical significance was probed using one-way ANOVA, $\mathrm{p}<0.05$ considered as significant.

\section{Results}

Data recorded for this research were obtained from 46 separate auditory cortical networks with an average of $47 \pm 4$ active neurons (range: 10-124 neurons) and an average age of $31 \pm 2$ days in vitro 
(range: 21-96 days in vitro). The in vitro networks were all spontaneously active. In order to maintain standard medium conditions, all cultures were subjected to a full medium change with the DMEM stock solution (no serum). Following stable recordings - determined by level plateaus of activity parameters per minute - the activity recorded in DMEM stock, referred to as "reference activity" (Ref), was monitored for a minimum of $30 \mathrm{~min}$. This period was followed by the addition of PTZ to induce rapid neuronal firing in the neuronal networks to mimic electrical activity that may occur in tinnitus. Once the PTZ-induced activity stabilized, one of the four experimental tinnitus drugs was added in successive steps to modulate the ongoing neuronal activity.

\subsection{Effects of pentylenetetrazol on in vitro cultures}

Typical responses to sequential addition of PTZ are shown in Fig. 1A. This 28 day in vitro auditory cortical network showed a gradual increase from reference (Ref) in spike rate and burst rate from 0.1 to $0.9 \mathrm{mM}$ concentration of PTZ, reaching maximum plateau at $1.0 \mathrm{mM}$. Concentrations $(1.1-1.5 \mathrm{mM})$ of PTZ higher than $1.0 \mathrm{mM}$ decreased the spike and burst rates. Each data point on the $Y$ axis reflects the "minute mean" for spike and burst values averaged over 46 active units in the culture. While the application of $200 \mu \mathrm{M} \mathrm{PTZ}$ at 62 min (Fig. 1A) showed a sudden increase in spike rate for the initial moment of drug application, this is interpreted as an artifact due to injection of eddy currents for the pipette and unequal distribution of PTZ, which slowly stabilized as the culture bath reached uniform concentration. In most experiments, the interval between drug injections was approximately $30 \mathrm{~min}$, allowing for at least $15 \mathrm{~min}$ of stable activity. Based on these data $(n=6)$, PTZ of $1.0 \mathrm{mM}$ was determined to maximally induce excitation of neurons in the cultures (Fig. 1B). Hence, $1.0 \mathrm{mM}$ PTZ was used as the standard concentration in all subsequent experiments. The auditory cortical network exhibited an average spike rate increase of $139.6 \pm 27 \%$, and an average burst rate increase of $129.7 \pm 28 \%$ under $1.0 \mathrm{mM} \mathrm{PTZ} \mathrm{(} \mathrm{n}=39$; Fig. 1B). Activity increase under PTZ is persistent and remained stable for more than $8 \mathrm{~h}$, and is reversible, i.e. activity returns to the reference level upon medium changes (data not shown). To identify if there were any morphological changes of the neurons with application of PTZ, neurons and neuronal processes were visually monitored throughout the experiment (Fig. 1C). We found no overt changes in morphology with $1.0 \mathrm{mM}$ PTZ. In all experiments, the action potential waveforms were monitored also during sequential application of PTZ. No statistical significance was found at PTZ of $100 \mu \mathrm{M}, 500 \mu \mathrm{M}, 1.0 \mathrm{mM}$, or $1.5 \mathrm{mM}$ (ANOVA, P>0.05).

\subsection{Effects of potassium channel blocker linopirdine on PTZ-treated cultures}

In the presence of $1.0 \mathrm{mM} \mathrm{PTZ,} \mathrm{various} \mathrm{concentrations} \mathrm{of}$ linopirdine were applied. Linopirdine is a potent inhibitor of the excitation by PTZ, displaying an effect at the micromolar concentration range. All neuronal activity ceased at $500 \mu \mathrm{M}$, but was reversible with a wash which consisted of a full medium change with fresh DMEM stock (Fig. 2A). Marked horizontal lines depict $>20$ min of stable activity following each application; these data points were taken into account for the calculation of the concentration-response curve. The $\mathrm{EC}_{50}$ of linopirdine is $176 \pm 7.0 \mu \mathrm{M}(\mathrm{n}=7$, Hill coefficient $=1.9$ ) calculated for seven experiments (Fig. 3). Raster plots seen in Fig. 2B display reference, $1.0 \mathrm{mM}$ PTZ and $150 \mu \mathrm{M}$ linopirdine conditions. PTZ increased spike production and burst coordination. Linopirdine $(150 \mu \mathrm{M})$ reduced the PTZ-induced excitation with no overt changes in neuronal morphology (Fig. 2C). Fig. 2D displays action potential waveforms under each of the experimental condition. No statistical significance was found among all experimental conditions (ANOVA, $\mathrm{P}>0.05$ ).

\subsection{Effects of the antioxidant L-carnitine on PTZ-treated cultures}

L-Carnitine is an over-the-counter antioxidant supplement which was used here to determine its effects on PTZ-treated cultures. Fig. 4A shows a typical PTZ treated culture responding to various concentrations
A

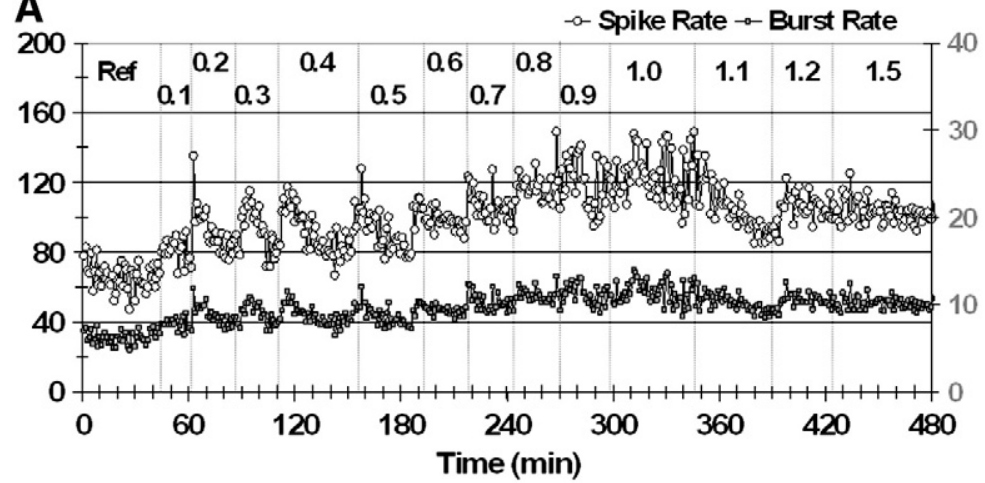

C

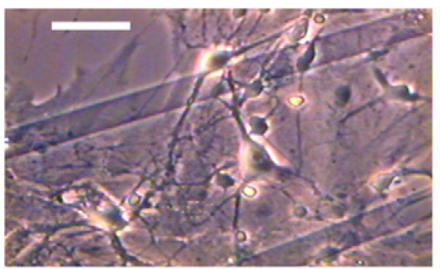

Ref

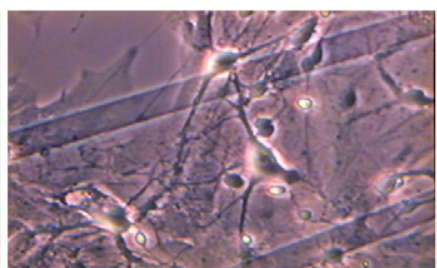

$1.0 \mathrm{mM}$ PTZ
B

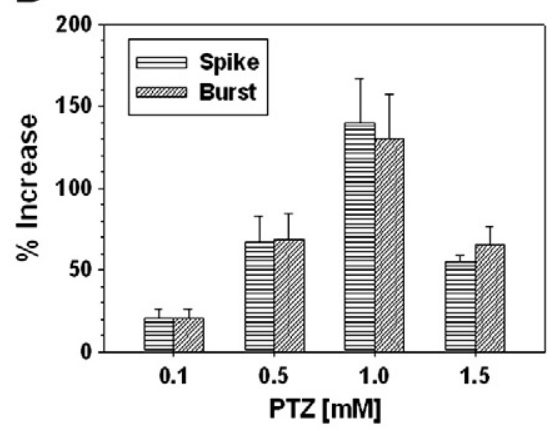

D

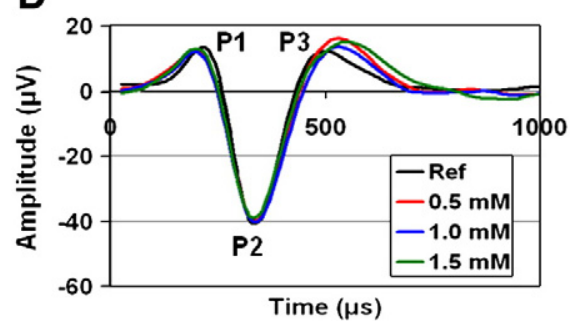

Fig. 1. (A) Typical response of auditory cortical network to increasing concentration of PTZ [mM]. Each data point represents spike count (left y-axis) and burst count (right y-axis) averaged across all units $(n=40)$ per minute. (B) Percent increase of spike rate and burst rate under $0.1 \mathrm{mM}(\mathrm{n}=8), 0.5 \mathrm{mM}(\mathrm{n}=11), 1.0 \mathrm{mM}(\mathrm{n}=6)$, and $1.5 \mathrm{mM}(\mathrm{n}=5)$ PTZ. (C) Cell morphology under phase-contrast microscopy (bar $=40 \mu \mathrm{m})$. (D) Single unit action potential waveforms averaged under each experimental condition $(\mathrm{n}=20$ each). 
A

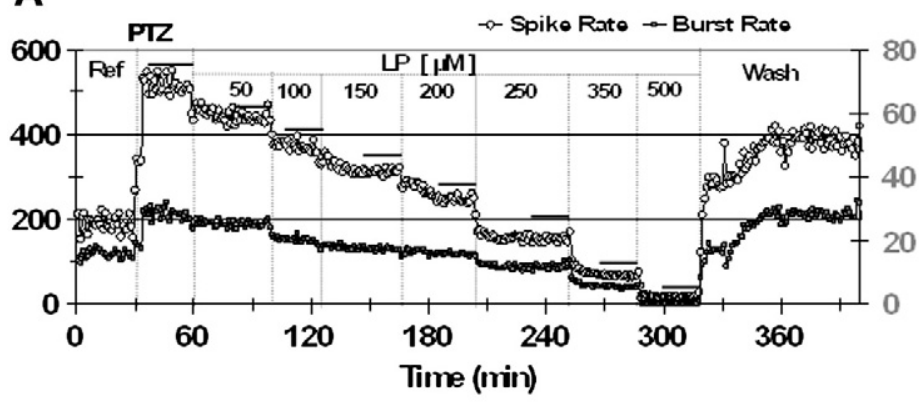

C

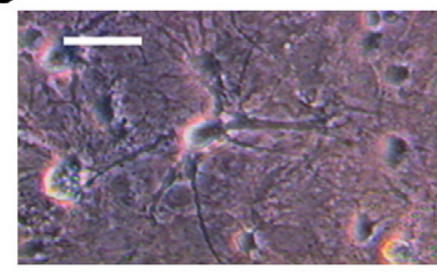

Ref

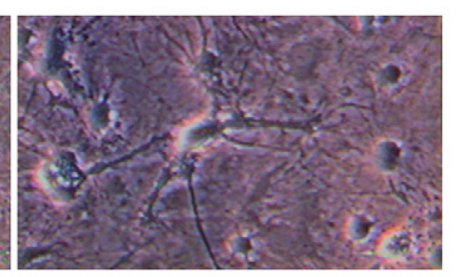

$150 \mu \mathrm{M}$ LP
B

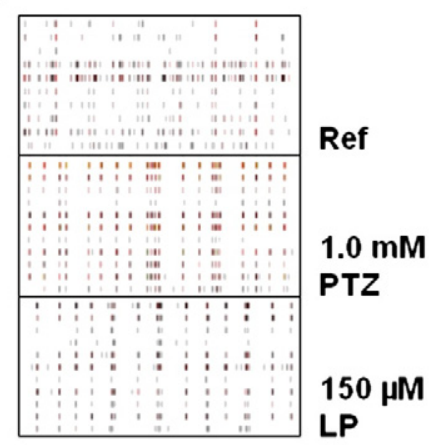

D

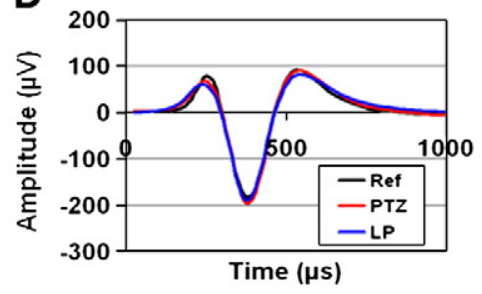

Fig. 2. (A) Response of auditory cortical network to increasing concentrations of linopirdine (LP). Each data point represents spike count (left y-axis) and burst count (right y-axis) averaged across all units $(n=20)$ per minute. Horizontal bars identify regions of stabilized network activity used for concentration-response calculation. (B) Raster plots (spike sequence) display of spontaneous activity. (C) Cell morphology under phase-contrast microscopy (bar $=40 \mu \mathrm{m}$ ). (D) Action potential waveforms under reference, 1.0 mM PTZ, and $150 \mu \mathrm{M}$ linopirdine.

of L-carnitine. L-Carnitine first became effective in reducing the PTZevoked activity at a level of $500 \mu \mathrm{M}$. At $2.0 \mathrm{mM}$, the activity was reduced to more than $50 \%$ of the reference level; a full medium change after applications of the highest dose brought the activity back to the native reference level. Fig. 3 indicates that the $\mathrm{EC}_{50}$ of $\mathrm{L}$-carnitine was $1569 \mu \mathrm{M} \pm 41 \mu \mathrm{M}(\mathrm{n}=8$; Hill coefficient $=1.2)$. Raster plots for L-carnitine application are depicted in Fig. 4B. No overt morphological changes (Fig. 4C) or action potential waveform changes (Fig. 4D) were noticed with the application of L-carnitine.

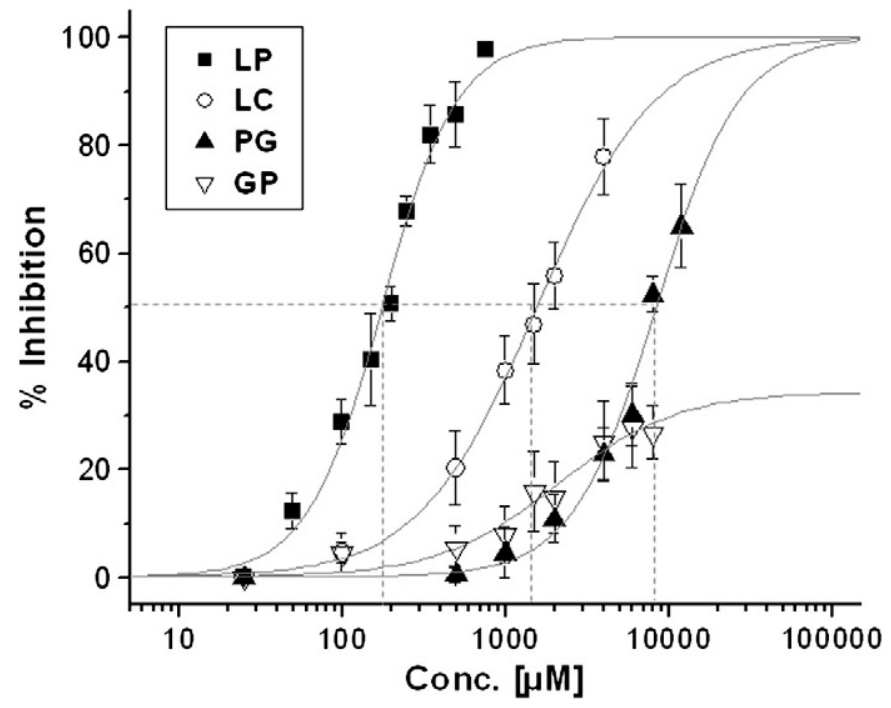

Fig. 3. Concentration-response curves for auditory cortical network pre-treated with 1.0 mM PTZ followed by application of linopirdine ( $\mathrm{LP} ; \mathrm{n}=7$, black squares), $\mathrm{L}$-carnitine (LC; $\mathrm{n}=8$, white circles), pregabalin (PG; $\mathrm{n}=8$, black upward triangles) and gabapentin (GP; $\mathrm{n}=8$, white downward triangles). The horizontal dashed line equals the $50 \%$ point of inhibition.

\subsection{Effects of the anticonvulsants gabapentin and pregabalin on} PTZ-treated cultures

Gabapentin (Neurontin) was not a potent inhibitor of PTZ-induced activity. No effect is seen at a concentration less than $4.0 \mathrm{mM}$ (Fig. 5A), far beyond the physiological range. The spike rate approached to the pre-PTZ level at the highest concentration tested $(12 \mathrm{mM})$. The concentration-response curve indicated that the efficacy of gabapentin is only $34.2 \% \pm 7.5$, thus, the $\mathrm{EC}_{50}$ is $2091 \pm 980 \mu \mathrm{M}$ (Hill slope $=1.2$; Fig. 3). At $2.0 \mathrm{mM}$, gabapentin decreased burst frequency without changing the overall burst pattern, which partly reflects its low efficacy (Fig. 5B). Neuronal morphology as well as action potential waveforms were unaffected by the application of gabapentin (Fig. 5C-D). Even at the high dose of $12 \mathrm{mM}$, a change in neuronal morphology was not discernible (data not shown).

The dose-dependent activity plot of pregabalin (Lyrica) follows a very similar pattern of gabapentin, its precursor, as the effect can only be seen at a concentration greater than a $2.0 \mathrm{mM}$ (Fig. 6A). Pregabalin, although ineffective in attenuating PTZ induced hyperactivity, shows $100 \%$ efficacy with an $\mathrm{EC}_{50}$ of $8360 \pm 307 \mu \mathrm{M}$ (Hill slope $=1.7$ ). The raster display shows that pregabalin still maintains a coordinated burst pattern initiated by PTZ. Pregabalin of $8.0 \mathrm{mM}$ also does not change cell morphology (Fig. 6C) or action potential waveforms (Fig. 6D) over the $4 \mathrm{~h}$ experimental time period.

\section{Discussion}

The effectiveness of the various tested compounds with the potential of eliminating tinnitus was linopirdine $>$ L-carnitine $>$ pregabalin $>$ gabapentin. Other channel antagonists were not tested since there have been a myriad of off-labeled drugs such as the GABA agonists and NMDA channel blockers used in the investigation of tinnitus (Akula et al., 2009; Elgoyhen and Langguth, 2009) that, for the most part, have proven ineffective. The fact that linopirdine (a potent blocker of the KCNQ4 potassium channel that is expressed in the mouse) was taken off the market for lack of efficacy in improving memory in Alzheimer patients, perhaps other KCNQx antagonists may be useful in this 
A

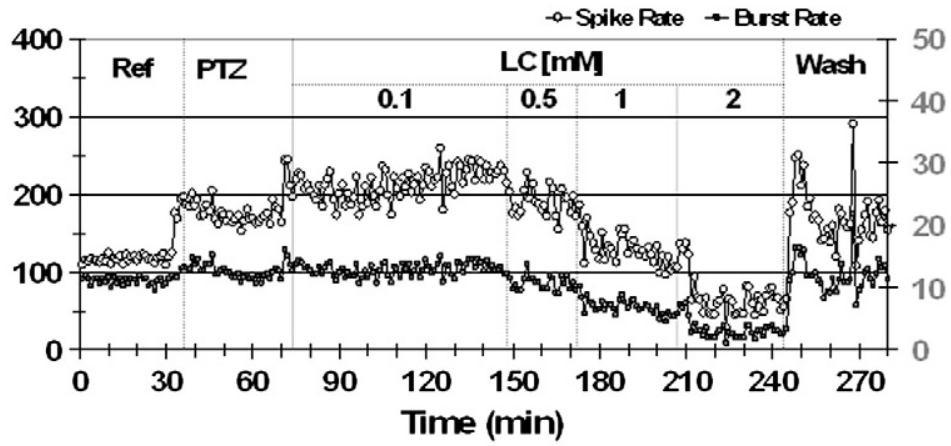

C

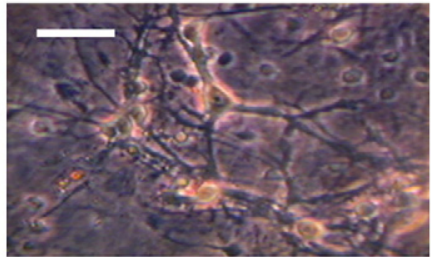

Ref

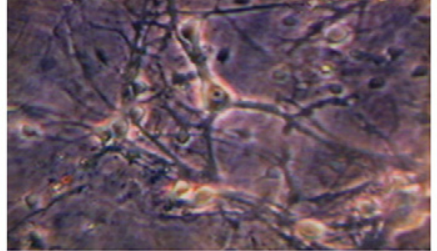

$1.5 \mathrm{mM}$ LC
B

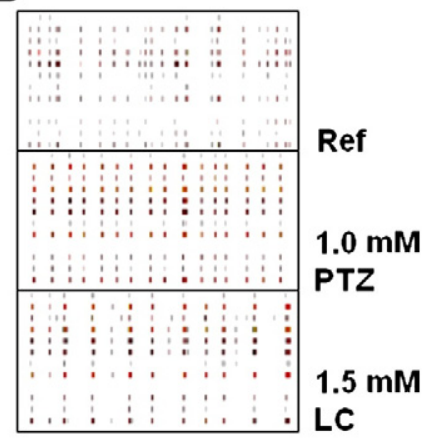

D

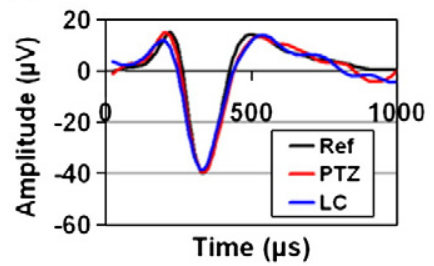

Fig. 4. (A) Response of auditory cortical network to increasing concentrations of L-carnitine (LC). Each data point represents spike count (left y-axis) and burst count (right y-axis) averaged across all units $(\mathrm{n}=20)$ per minute. (B) Raster plots (spike sequence) display of spontaneous activity. (C) Cell morphology under phase-contrast microscopy (bar $=40 \mu \mathrm{m}$ ). (D) Action potential waveforms under reference, $1.0 \mathrm{mM}$ PTZ, and $1.5 \mathrm{mM}$ L-carnitine.

instance. Linopirdine's side effects may be due to the inhibition of acetylcholine receptors (Berni et al., 2008) so that with other $\mathrm{K}^{+}$channel antagonists, there may be fewer "off target" effects that may be active.

Linopirdine is a potent $\mathrm{K}^{+}$channel blocker of the KCNQ4 sub-type and shows its greatest effect on the M-channel (Liang et al., 2005). The $\mathrm{M}$-channel has been implicated also in the neurogenesis of tinnitus (Holt et al., 2007). In the current study the $\mathrm{EC}_{50}$ of linopirdine was $176 \mu \mathrm{M}$, which is approaching a physiological range and may thus serve as an adjunctive therapy for tinnitus, but would have to be made available once again to the consumer market. The fact that linopirdine is classified also as a nootropic drug ("smart drug") and is surmised to work by altering the availability of neuronal neurochemicals. This may in fact improve neuronal oxygen supply. However, the efficacy of various nootropic substances in modulating tinnitus remains to be elucidated.

L-Carnitine is an over-the-counter supplement that is classified as an anti-oxidant. Its mode of action is to act as a scavenger of reactive oxygen species, a possible cause of tinnitus in patients (Berni et al.,
A

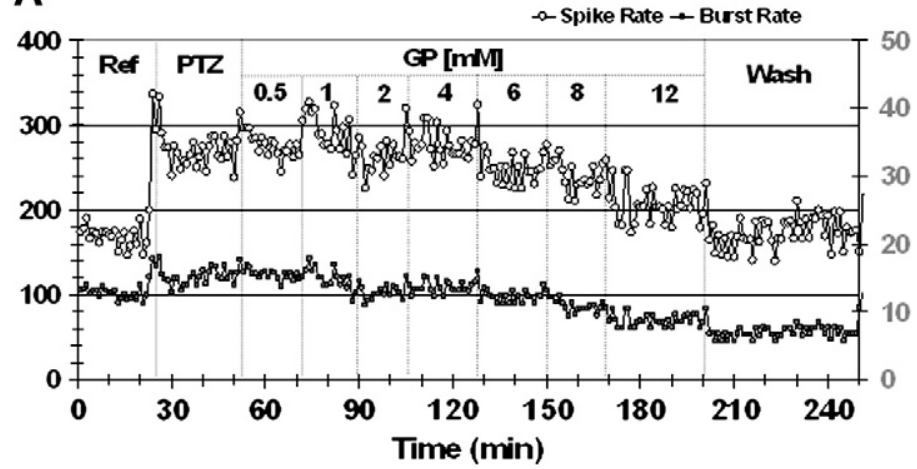

C

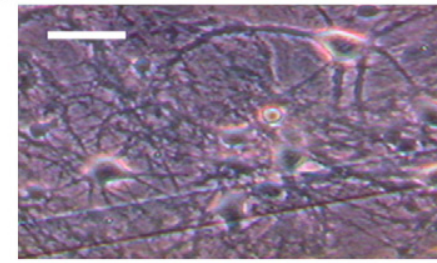

Ref
B

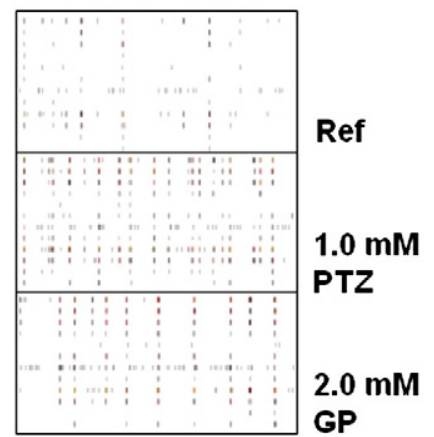

D

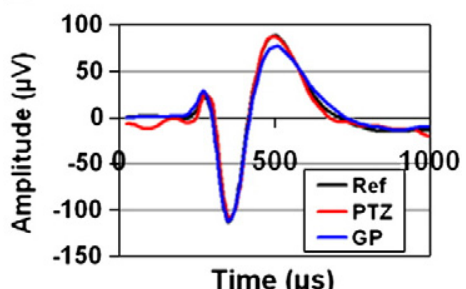

Fig. 5. (A) Response of auditory cortical network to increasing concentrations of gabapentin (GP). Each data point represents spike count (left y-axis) and burst count (right y-axis) averaged across all units $(n=87)$ per minute. (B) Raster plots (spike sequence) display of spontaneous activity. (C) Cell morphology under phase-contrast microscopy

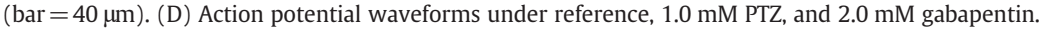


A

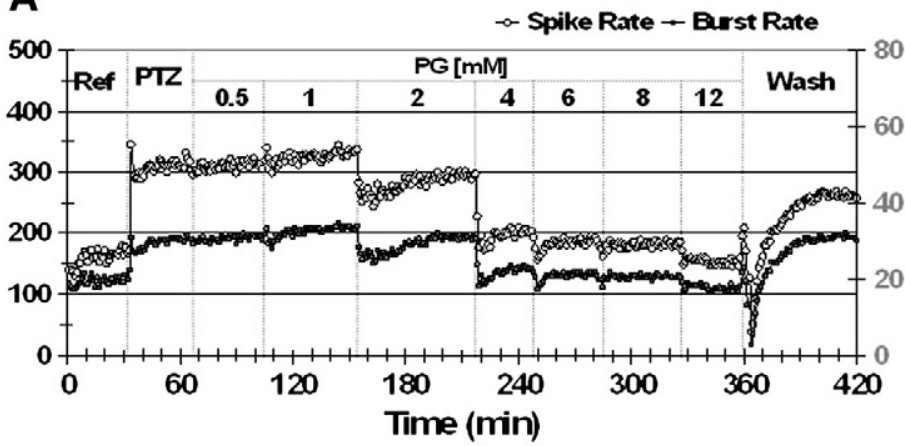

C

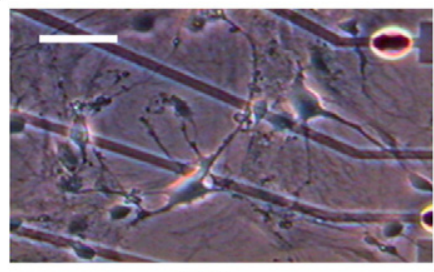

Ref

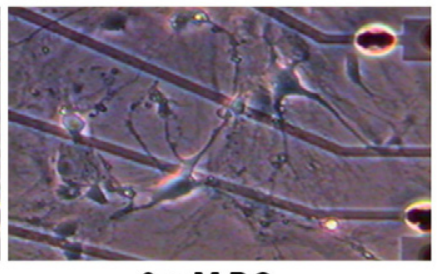

8 mM PG
B

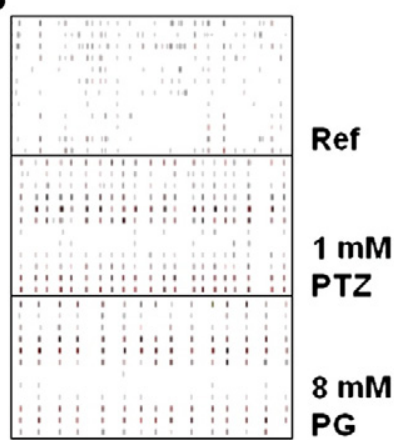

D

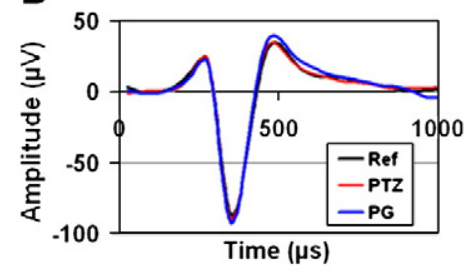

Fig. 6. (A) Response of auditory cortical network to increasing concentrations of pregabalin (PG). Each data point represents spike count (left y-axis) and burst count (right y-axis) averaged across all units $(\mathrm{n}=78)$ per minute. (B) Raster plots (spike sequence) display of spontaneous activity. (C) Cell morphology under phase-contrast microscopy

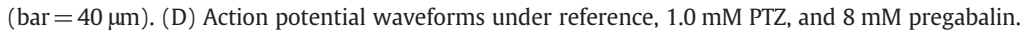

2008). However, the lack of effect of most over-the-counter supplements in treating tinnitus patients (Enrico et al., 2007) suggests that the mechanism of action of such agents is more complicated than previously considered. It is noted that the claims for this over the counter drug are touted as an effective treatment for tinnitus in the lay literature, and anecdotal claims are equally positive. Carnitine is actually an essential nutrient found in the body that helps in transporting fat into mitochondria where it is transformed into energy in the form of ATP. While carnitine exists in three forms: L-carnitine (the most recommended form), acetyl-L-carnitine (recommended for certain Alzheimer patients), and propionyl-L-carnitine (recommended for certain types of heart disease), we used only the L-isomer in our studies as the former is the more active form of the drug than the D-isomer type. We have not found any definitive studies that have used the other forms of the supplement in the treatment of tinnitus.

Pregabalin and gabapentin both bind to the $\mathrm{Ca}^{++}$channel subunit $\alpha_{2}-\delta$ (Taylor et al., 2007). Pregabalin was more potent at a lower concentration than gabapentin in reducing the ongoing electrical activity induced by PTZ, albeit, at a rather high concentration. Pregabalin and gabapentin are structurally similar, but pregabalin has greater efficacy at smaller dosages in reducing certain types of pain and reducing the effects of electrical discharges in epilepsy patients. Thus, if epilepsy or pain can serve as homologue of tinnitus, these drugs may work in modulating tinnitus. Pregabalin and gabapentin were originally developed as GABA receptor antagonists but they are weakly active at this site. The high $\mathrm{EC}_{50}$ that we observed with PTZ-induced auditory cortical networks is consistent with these drugs directly antagonizing the effects of PTZ. It has been reported that gabapentin does not bind to the $G_{A B A}$ receptor, thus, this might perhaps account for its less potential efficacy for tinnitus patients (Shimizu et al., 2004). Anecdotal reports of patients on a regimen of pregabalin for the management of pain reported an exacerbation of their tinnitus, but some patients reported also reduced tinnitus as a result of taking pregabalin. Thus, our in vitro results might suggest that a more effective type of Ca-channel antagonist may be necessary to treat tinnitus.

There is emerging evidence to suggest that dysregulation within the auditory system after insult may underlie aberrant excitation in the form of tinnitus (Hwang et al., 2011a,b). These investigators found altered expression of COX-2 and NMDA receptor genes in the cochlea and midbrain in salicylate-induced tinnitus (Noreña et al., 2010). Hwang and his colleagues induced the "tinnitus-like activity" in a mouse model trained to experience tinnitus. There is evidence to suggest also that the pathophysiology underlying tinnitus may be mediated via receptors or ion channels of the NMDA or GABA receptor type (Hansen et al., 2004), chloride channel types or even auditory efferent pathway neuroactive substances (Puel, 1995). This may be manifested at multiple levels of the auditory neuraxis to include not only the hair cells of the cochlea, but brain stem auditory structures such as the dorsal cochlear nucleus (Kaltenbach, 2006), inferior colliculus (Melcher et al., 2000), auditory cortex (Eggermont, 2008), as well as non-auditory CNS structures such as the limbic system (Lockwood et al., 1998). While our data was obtained in vitro and thus would not reveal any information about the auditory system loci, nevertheless, further studies to reveal precisely anatomical loci are worthy of pursuit.

\section{Conclusion}

In conclusion, we used PTZ, a proconvulsant agent that causes excitation of cortical neurons without inducing cell necrosis or apoptosis. Previous studies have used either salicylates or quinine to induce similar excitatory activity. Drugs of the $\mathrm{K}^{+}$channel blocker type, the $\mathrm{Ca}^{2+}$ channel antagonists, as well as an antioxidant were used to attenuate the effects of PTZ. We found a protective effect from neuronal excitation under those conditions that modulated or blocked neuronal electrical activity at the 50\% (ED) level. Most of the drug levels, however, were beyond ideal physiological range, except linopirdine, and thus clinical use of these drugs may cause unwanted side effects. The MEA platform therefore serves as a useful vehicle for screening drugs (i.e., assessing potential toxicity and/or safety) that may reduce the percept of tinnitus. This study fulfills our proof of principle for testing the efficacy of thousands of FDA drugs already available on the market that might attenuate increased ongoing electrical activity of mouse auditory cortical neurons in an experimental condition resembling tinnitus. 


\section{Acknowledgment}

This research was supported in part by a Faculty Research Grant (GA9289) from UNT awarded to EJM, and the Charles Bowen Endowment to the Center for Network Neuroscience. Pregabalin was a kind gift from Pfizer. We thank Timothy Jaquez for help with the pilot data experiments. We are grateful to Dr. Maryam Parviz for a critical review of the manuscript.

\section{References}

Akula, K.K., Dhir, A., Kulkarni, S.K., 2009. Effect of various antiepileptic drugs in pentylenetetrazol-induced seizure model in mice. Methods Find. Exp. Clin. Pharmacol. 31, 423-432.

Baguley, D.M., 2002. Mechanisms of tinnitus. Br. Med. Bull. 63, 195-212.

Berni, A., Meschini, R., Filippi, S., Palitti, F., De Amicis, A., Chessa, L., 2008. L-Carnitine enhances resistance to oxidative stress by reducing DNA damage in Ataxia telangiectasia cells. Mutat. Res. 650, 165-174.

Boguski, M.S., Mandl, K.D., Sukhatme, V.P., 2009. Drug discovery. Repurposing with a difference. Science 324, 1394-1395.

Denk, D.M., Heinzl, H., Frank, P., Ehrenberger, K., 1997. Caroverine in tinnitus treatment. A placebo-controlled blind study. Acta Otolaryngol. 117, 825-830.

Eggermont, J.J., 2008. Role of the auditory cortex in noise- and drug-induced tinnitus. Am. J. Audiol. 172, 162-169.

Elgoyhen, A.B., Langguth, B., 2009. Pharmacological approaches to the treatment of tinnitus. Drug Discov. Today 15, 300-305.

Enrico, P., Sirca, D., Mereu, M., 2007. Antioxidants, minerals, vitamins, and herbal remedies in tinnitus therapy. Prog. Brain Res. 166, 323-330.

Gopal, K.V., Gross, G.W., 1996. Auditory cortical neurons in vitro: cell culture and multichannel extracellular recording. Acta Otolaryngol. 116, 690-696.

Gopal, K.V., Gross, G.W., 2004. Unique responses of auditory cortex neurons in vitro to low concentrations of quinine. Hear. Res. 192, 10-22.

Gopal, K.V., Miller, B.R., Gross, G.W., 2007. Acute and sub-chronic functional neurotoxicity of methylphenidate on neural networks in vitro. J. Neural Transm. $114,1365-1375$

Hansen, S.L., Sperling, B.B., Sánchez, C., 2004. Anticonvulsant and antiepileptogenic effects of GABAa receptor ligands in pentylenetetrazole-kindled mice. Prog. Neuropsychopharmacol. Biol. Psychiatry 28, 105-113.

Holt, J.R., Stauffer, E.A., Abraham, D., Géléoc, G.S., 2007. Dominant-negative inhibition of M-like potassium conductances in hair cells of the mouse inner ear. J. Neurosci. 27 8940-8951.

Hwang, J.H., Chen, J.C., Yang, S.Y., Wang, M.F., Liu, T.C., Chan, Y.C., 2011a. Expression of COX-2 and NMDA receptor genes at the cochlea and midbrain in salicylate-induced tinnitus. Laryngoscope 121, 361-364.

Hwang, J.H., Chen, J.C., Yang, S.Y., Wang, M.F., Liu, T.C., Chan, Y.C., 2011b. Expression of tumor necrosis factor-alpha and interleukin-1 beta genes at the cochlea and the inferior colliculus in salicylate-induced tinnitus. J. Neuroinflammation 8, 30 (Apr 9).

Jastreboff, P.J., 1990. Phantom auditory perception (tinnitus): mechanisms of generation and perception. Neurosci. Res. 8, 221-254.

Jung, T.T., Rhee, C.K., Lee, C.S., Park, Y.S., Choi, D.C., 1993. Ototoxicity of salicylate, nonsteroidal anti-inflammatory drugs, and quinine. Otolaryngol. Clin. North Am. 26, 791-810.
Kaltenbach, J.A., 2000. Neurophysiologic mechanisms of tinnitus. J. Am. Acad. Audiol. $11,125-137$.

Kaltenbach, J.A., 2006. Summary of evidence pointing to a role of the dorsal cochlear nucleus in the etiology of tinnitus. Acta Otolaryngol. Suppl. 556, 20-26.

Kay, I.S., Davies, W.E., 1993. The effect of nimodipine on salicylate ototoxicity in the rat as revealed by the auditory evoked brain-stem response. Eur. Arch. Otorhinolaryngol. 250, 51-54.

Keefer, E.W., Gramowski, A., Stenger, D.A., Pancrazio, J.J., Gross, G.W., 2001 Characterization of acute neurotoxic effects of trimethylolpropane phosphate vic neuronal network biosensors. Biosens. Bioelectron. 16, 513-525.

Kenmochi, K., Eggermont, J.J., 1997. Salicylate and quinine affect the central nervous system. Hear. Res. 113, 110-116.

Klöcker, N., Musshoff, U., Madeja, M., Speckmann, E.J., 1996. Activation of ATP-sensitive potassium channels in follicle-enclosed xenopus oocytes by the epileptogenic agent pentylenetetrazol. Pflugers Arch. 431, 736-740.

Lanting, C.P., de Kleine, E., van Dijk, P., 2009. Neural activity underlying tinnitus generation: results from PET and fMRI. Hear. Res. 255, 1-13.

Liang, G., Moore, E.J., Ulfendahl, M., Rydqvist, B., Järlebark, L., 2005. An M-like potassium current in the guinea pig cochlea. ORL J Otorhinolaryngol. Relat. Spec. 67, 75-82.

Littler, T.S., 1965. The Physics of the Ear. Pergamon Press, Oxford, UK.

Lockwood, A.H., Salvi, R.J., Coad, M.L., Towsley, M.L., Wack, D.S., Murphy, B.W., 1998 The functional neuroanatomy of tinnitus: evidence for limbic system links and neural plasticity. Neurology 50, 114-120.

Lockwood, A.H., Salvi, R.J., Burkard, R.F., Galantowicz, P.J., Coad, M.L., Wack, D.S., 1999. Neuroanatomy of tinnitus. Scand. Audiol. Suppl. 51, 47-52.

Lockwood, A.H., Salvi, R.J., Burkard, R.F., 2002. Tinnitus. N. Engl. J. Med. 347, 904-910.

Melcher, J.R., Sigalovsky, I.S., Guinan, J.J., Levine, R.A., 2000. Lateralized tinnitus studied with functional magnetic resonance imaging: abnormal inferior colliculus activation. J. Neurophysiol. 83, 1058-1072.

Morefield, S.I., Keefer, E.W., Chapman, K.D., Gross, G.W., 2000. Drug evaluations using neuronal networks cultured on microelectrode arrays. Biosens. Bioelectron. 15, 383-396.

Mühlau, M., Rauschecker, J.P., Oestreicher, E., Gaser, C., Röttinger, M., Wohlschläger, A M., Simon, F., Etgen, T., Conrad, B., Sander, D., 2006. Structural brain changes in tinnitus. Cereb. Cortex 16, 1283-1288.

Noreña, A.J., Moffat, G., Blanc, J.L., Pezard, L., Cazals, Y., 2010. Neural changes in the auditory cortex of awake guinea pigs after two tinnitus inducers: salicylate and acoustic trauma. Neuroscience 166, 1194-1209.

Passe, E.R.G., 1951. Sympathectomy in relation to Ménière's disease, nerve deafness and tinnitus. A report on 110 cases. Proc. R. Soc. Med. 44, 760-772.

Puel, J.-L., 1995. Chemical synaptic transmission in the cochlea. Prog. Neurobiol. 47, 449-476.

Shimizu, S., Honda, M. Tanabe, M., Ono, H., 2004. GABA receptors do not mediate the inhibitory actions of gabapentin on the spinal reflex in rats. J. Pharmacol. Sci. 96 444-449.

Taylor, C.P., Angelotti, T., Fauman, E., 2007. Pharmacology and mechanism of action of pregabalin: the calcium channel $\alpha_{2}-\delta$ (alpha2-delta) subunit as a target for antiepileptic drug discovery. Epilepsy Res. 73, 137-150.

Thabet, E.M., 2009. Evaluation of tinnitus patients with normal hearing sensitivity using TEOAEs and TEN test. Auris Nasus Larynx 36, 633-636.

Tobinick, E.L., 2009. The value of drug repositioning in the current pharmaceutical market. Drug News Perspect. 22, 119-125.

Zenner, H.P., Ernst, A., 2003. Cochlear-motor, transduction and signal-transfer tinnitus: models for three types of cochlear tinnitus. Eur. Arch. Otorhinolaryngol. 249, 447-454 